\title{
Quantification of Skin Marker Movement at the Malleoli and Talar Heads
}

\author{
Ivan Birch, PhD* \\ Kevin Deschamps, MSc†
}

\begin{abstract}
Background: Quantifying subtalar joint kinematics during locomotion is a major challenge but is critical to understanding foot function. The difficulty of modeling the subtalar joint is demonstrated by the plethora of three-dimensional multisegment foot models lacking specific consideration of the subtalar joint. Scientific attempts to develop an adequate method of quantifying subtalar joint kinematics should include investigation of the movement of skin-mounted markers. This study reports on a single-subject investigation into this topic.
\end{abstract}

Methods: Radiopaque markers were attached to the skin overlying the medial and lateral malleoli and the medial and lateral talar heads of a single subject. Frontal, sagittal, and transverse plane radiographs were taken with the foot in the fully pronated and fully supinated positions. Parallax corrected measurements were taken of the displacement of the markers from the bony landmarks. Measurements were also taken of the effect of these displacements on angular calculations.

Results: Skin movement at the four anatomical locations was not uniform, with displacements varying from 0.61 to $22.18 \mathrm{~mm}$. Movement of the malleolar markers was found to be less than that of the talar head markers. The distortion of angular measurements caused by movement of the skin markers relative to the bony landmarks was found to be only $1^{\circ}$ in the sagittal and transverse planes and $5^{\circ}$ in the frontal plane.

Conclusions: For this subject, skin-mounted markers could be used to assess subtalar joint motion. Further studies are necessary to investigate the implications of these findings to the wider population. (J Am Podiatr Med Assoc 101(6): 497-504, 2011)

During the past two decades, a plethora of threedimensional (3-D) multisegment foot models have been published. ${ }^{1,2}$ These models provide the clinician with information on the kinematics of specific foot segments during locomotion and are a considerable improvement over the previous singlesegment foot models. ${ }^{3,4}$ However, these 3-D multisegment foot models have some weaknesses, particularly related to the difficulties of locating specific bones in the foot and the potential effects of skin movement artifact. ${ }^{5-8}$ A good example of these problems can be seen in relation to the subtalar

*Faculty of Health and Human Sciences, University of West London, Brentford, England.

$\dagger$ Division of Musculoskeletal Disorders, University Hospitals Leuven, K.U. Leuven, Leuven, Belgium; Research Center for Musculoskeletal Rehabilitation, Department of Rehabilitation Sciences, K.U. Leuven, Leuven, Belgium.

Corresponding author: Ivan Birch, PhD, Pro Dean, Faculty of Health and Human Sciences, University of West London, Paragon House, Boston Manor Road, Brentford, Middlesex, TW89GA England. (E-mail: Ivan.Birch@tvu. ac.uk) joint, where their effects have resulted in there currently being no noninvasive, in vivo method of measuring the joint's motion during walking.

Skin movement has always been a problem for human motion analysis. ${ }^{9-14}$ Any optical system used to assess the relative movement of limb segments requires the use of marks made directly on the skin of the subject or the attachment of markers. In both cases, although it is the movement of the whole segment, usually thought of as being that of the underlying bone, that is under investigation, what is actually produced is an assessment of the movement of the overlying skin.

Skin is a complex structure that varies in nature and mechanical behavior from person to person. ${ }^{9,10}$ Furthermore, the behavior of skin in terms of the degree to which it moves over the underlying bones is not constant for each individual but varies from anatomical location to anatomical location. In some areas, such as the thigh, the skin is relatively free to move over the bones, whereas in others, such as the 
heel, the skin is tightly bound to the underlying structures.

The significance of any error introduced to motion analysis by skin movement also depends on the measurement being made. ${ }^{15}$ The influence of skin movement on the measured movement is a function of the amount of skin movement relative to the total amount of movement of the segment under investigation. As a result, the measurements of large movements of large segments will tend to be less affected than will those of small movements of small segments.

Several authors have given consideration to the problem of skin movement during movement analysis. ${ }^{10,14,16-18}$ Regarding the ankle and subtalar joint, the articles by Westblad et $\mathrm{al}^{19}$ and Nester et $\mathrm{al}^{7}$ are of particular note. Westblad et al ${ }^{19}$ investigated the differences in ankle motion during the stance phase of walking as measured by skinmounted markers and bone-anchored markers. They found that the patterns of movement shown by the two methods were generally in agreement but that discrepancies between rotation magnitudes were "not negligible." Their results showed that the magnitudes demonstrated by the skin-mounted markers were, on the whole, greater than those of the bone-anchored markers. They concluded by saying that although skin-mounted markers provided good sagittal plane results, greater problems were posed by frontal and transverse plane results. The later study by Nester et al, ${ }^{7}$ comparing the use of skin-, plate-, and bone-mounted markers, concurred with the findings of Westblad et $\mathrm{al}^{19}$ in terms of general patterns of motion, reporting that the match between the kinematic data from skin-, plate-, and bone-mounted marker protocols was reasonable or good.

A crucial factor in developing a fully clinically relevant 3-D multisegment foot model for motion analysis is the ability to measure the motion occurring at the subtalar joint. The first stages in the development of such a model were described by Birch and Deschamps. ${ }^{20}$ The marker placement model developed for this work defined the talus and calcaneus as rigid bodies using eight markers. The calcaneus was defined using four markers at the following anatomical locations: posterior lateral calcaneus, posterior medial calcaneus, anterior lateral calcaneus, and anterior medial calcaneus (Fig. 1A). The talus was defined using the lateral and medial talar head markers and a midankle virtual marker (Fig. 1B) located midway between the medial and lateral malleolar markers. Although the markers located on the tightly bound skin of the calcaneus could be expected to represent the movement of the underlying bone with some degree of accuracy, those located on the talar head and the malleoli, where the skin is less tightly bound, were subject to an unknown amount of skin movement artifact. This article reports a single-subject investigation into the movement of skin-mounted markers relative to the medial and lateral heads of the talus and the medial and lateral malleoli. This work was undertaken as the second stage of a larger project to investigate the feasibility of developing a noninvasive in vivo method of assessing subtalar joint motion during walking.

\section{Methods}

In view of the ethical implications of undertaking nonessential radiograph investigations of recruited subjects, this work was performed using one of the investigators as the subject (age, 48 years; height, $1.75 \mathrm{~m}$; weight, $82 \mathrm{~kg}$; and body mass index [calculated as weight in kilograms divided by height in meters squared], 26.8). Appropriate ethics consent was obtained from the School of Health Professions Ethics Committee, University of Brighton, England.

The movement of the skin over the medial and lateral sides of the head of the talus and the medial and lateral malleoli was investigated using radiographic images. Nine radiopaque markers (small steel washers) were attached to the skin of the subject's right leg and foot using double-sided adhesive tape at the anatomical locations of the marker placement model. The markers were positioned by palpating the anatomical landmarks using a written protocol developed for the investigation. The markers were positioned with the foot semiweightbearing, with the posterior of the calcaneus and leg vertical.

The location of the washers was also marked using a permanent fiber-tipped pen. This marking of the skin subsequently allowed markers for the CODA 3-D movement analysis system (CODA MPX30; Charnwood Dynamics Ltd, Leicestershire, England) to be placed in the same positions. A series of six radiographs were subsequently taken (three planes, two positions). The first three radiographs were taken with the subtalar joint actively fully pronated by the subject, the second three with the subtalar joint actively fully supinated. For the frontal and transverse plane radiographs, the subject was seated facing the radiograph unit, and at right angles (left side closest) to the unit for the sagittal plane radiographs. The radiograph unit 

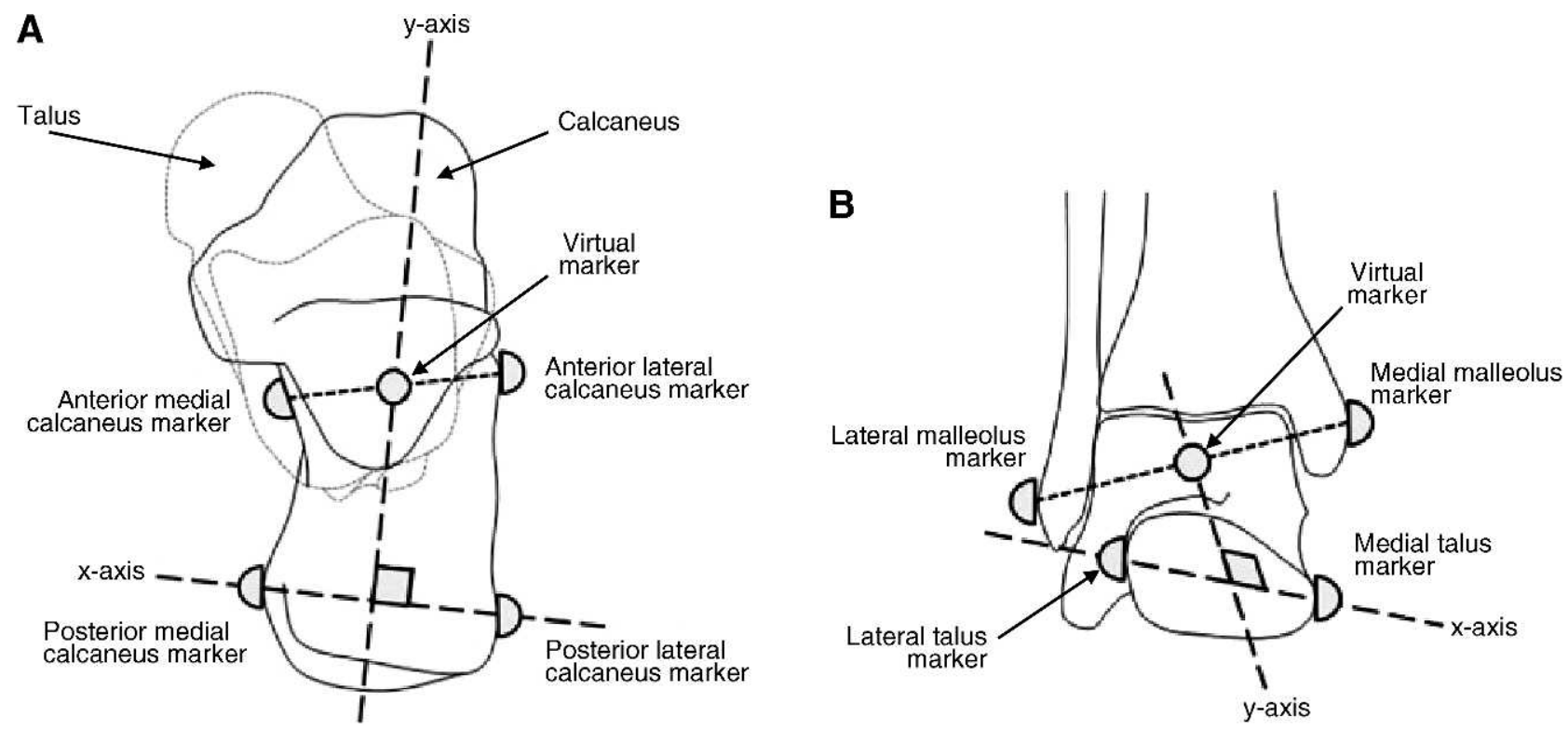

Figure 1. Marker placement at the calcaneus (A) and at the talus (B).

head was positioned $0.3 \mathrm{~m}$ from the ground with the face vertical for the frontal and sagittal plane radiographs, and facing downwards, parallel to the ground, for the transverse plane radiographs. Transverse plane alignment of the radiograph unit was achieved by positioning the radiograph unit anterior to the knee to remove the obscuring effect of the tibia and fibula. The plate was positioned $1.3 \mathrm{~m}$ from the radiograph unit head in all cases. For all of the radiographs, the right foot was held clear of the ground. A nonweightbearing position was used to facilitate the use of the full range of active motion at the subtalar joint, with tension being placed on the tendons by the muscles. In this way, the maximum possible degree of skin displacement was elicited.

Once the radiograph data had been collected, the fiber-tipped pen marks were used to locate the CODA markers. Placing the subject's leg facing one of the CODA scanners, two sets of data were then collected using the CODA MPX30 at $200 \mathrm{~Hz}$, one with the subtalar joint fully pronated and one with the subtalar joint fully supinated (Fig. 2). By careful positioning of the subject's leg, it was possible to align the CODA system planes and the anatomical planes of the subject's leg and foot. The subject's frontal plane corresponded to the CODA XZ plane, the sagittal to the $\mathrm{YZ}$, and the transverse to the $\mathrm{XY}$.

\section{Radiograph Orientation}

To validate the orientation of the subject's leg in relation to the radiograph unit, marker separations measured from the radiographs were correlated with those from the CODA. It was not anticipated that these two sets of measurements would be the same. The radiograph measurements were subject to a combination of the effects of parallax and rescaling due to physical distances between the radiograph unit, the leg and foot, and the radiograph plate. Nevertheless, the relationship between the magnitudes of the various marker separations derived from the radiographs and the CODA should show a strong correlation if the orientation of the leg and foot was similar when the two sets of data were collected.

Marker separations were derived from the CODA using the dynamic origin option in the CODA software and were exported into Microsoft Excel 97-2003 (Microsoft Corp, Redmond, Washington). The CODA's planar marker separations were converted to linear distances using basic trigonometry.

A horizontal radiograph viewer was used to establish the marker separation measurement from the radiographs. The center of each washer, which varied in shape from a circle to a straight line, was established using a Vernier caliper and marked. The marker separations were then measured using the same Vernier caliper. The repeatability of this protocol was assessed by measuring the same marker separation ten times, removing the washer center mark between each measurement. During this process, the scale of the Vernier was obscured so that the measurer could not see the outcome of each measurement, the measurement being record- 

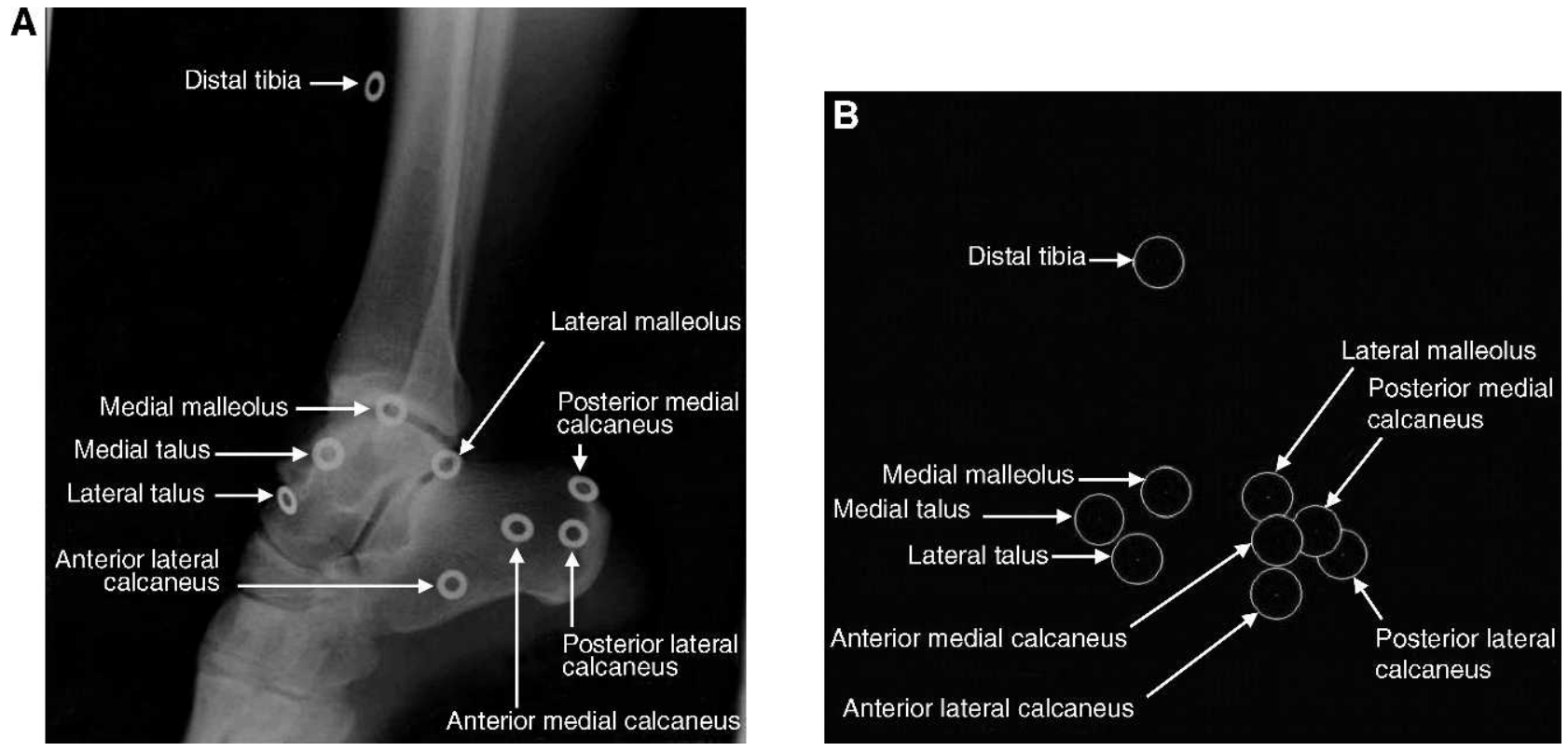

Figure 2. Examples of radiograph (A) and CODA (B) data showing marker locations.

ed by an independent second party. This data produced a mean $\pm \mathrm{SD}$ measurement of $63.8 \pm$ $0.094 \mathrm{~mm}$ and a coefficient of variation of $0.15 \%$. The measurement protocol was, therefore, accepted as being suitably repeatable for this investigation. The measurement protocol was then applied to each of the six radiographs; in each case, all possible marker separations were established. Note that measurements from the distal tibial marker could not be measured from the transverse plane radiographs owing to the opacity of the tibia. A correlation coefficient was calculated to establish the relationship between the CODA- and radiograph-derived data.

\section{Marker Displacement}

The locations of the medial and lateral heads of the talus and the medial and lateral malleoli were established on the radiographs, with the location of the marker centers having already been established. Measurement of the displacement of the markers from the bony landmarks, the location of which they were intended to represent, had a variety of elements to it. The displacement of each marker from the appropriate bony landmark was established using a series of three measurements, representing the displacement along each of the $\mathrm{x}-$, $\mathrm{y}$-, and z-axes. These measurements were taken from the frontal and sagittal plane radiographs, which had shown the better correlation and, therefore, the better planar orientation. The $y$ - and z-axis measure- ments were taken from the sagittal plane radiograph, and the x-axis displacement was taken from the frontal plane radiograph.

The orientation of the z-axis was established on the radiograph using an acetate sheet, on which a right-angled grid had been printed, that was placed over the radiograph on the viewer. The $\mathrm{z}$ - or vertical axis was determined by aligning the vertical lines of the grid with the long axis of the fibula. The fibula was used, rather than the tibia, because it was the fibula that was primarily used to determine the $\mathrm{z}$ axis when using the CODA. Once the vertical lines of the grid had been aligned with this long axis, the $\mathrm{x}$ - or y-axis (depending on which radiograph was under investigation) was determined by the horizontal lines of the grid. Displacement measurements were then taken parallel to the grid lines, producing an $\mathrm{x}, \mathrm{y}$, and $\mathrm{z}$ displacement for each bone/marker pairing.

In determining the direction of the displacement of a marker relative to the bony landmark along an axis, the following protocol was used ${ }^{21}$ :

positive $\mathrm{x}$-axis $=$ lateral; negative $\mathrm{x}$-axis $=$ medial positive $\mathrm{y}$-axis $=$ anterior; negative $\mathrm{y}$-axis $=$ posterior positive $\mathrm{z}$-axis $=$ proximal; negative $\mathrm{z}$-axis $=$ distal

Although the measurements given herein are in millimeters, they do not actually represent millimeters of movement in terms of the markers. These measurements were derived from radiographs that were not one-to-one scale representations of the 
foot. Furthermore, the degree to which the radiographs were not one-to-one scale representations of the bones varied from radiograph to radiograph.

To calculate the actual displacement, a correction factor was established for each measurement using the radiopaque markers, which were of a uniform shape (circular) and size ( $8.7 \mathrm{~mm}$ in diameter). The fact that they were circular meant that no matter what the orientation of the washer to the radiograph unit, the largest possible measurement of the washer from the radiograph would always represent the overall diameter of the washer as recorded by the radiograph.

Having established the size of each of the washers from the appropriate radiographs, a correction factor could be calculated by dividing the actual size of the washers $(8.7 \mathrm{~mm})$ by the size measured from the radiograph. The actual measurements of marker displacement taken from the frontal and sagittal plane radiographs were then corrected by multiplying them by the appropriate correction factor.

\section{Effect of Skin Movement on Angular Measurements}

Using the method with the radiograph viewer and the grid, it was possible to assess the orientation of each pair of markers when the foot was fully pronated and fully supinated relative to a constant

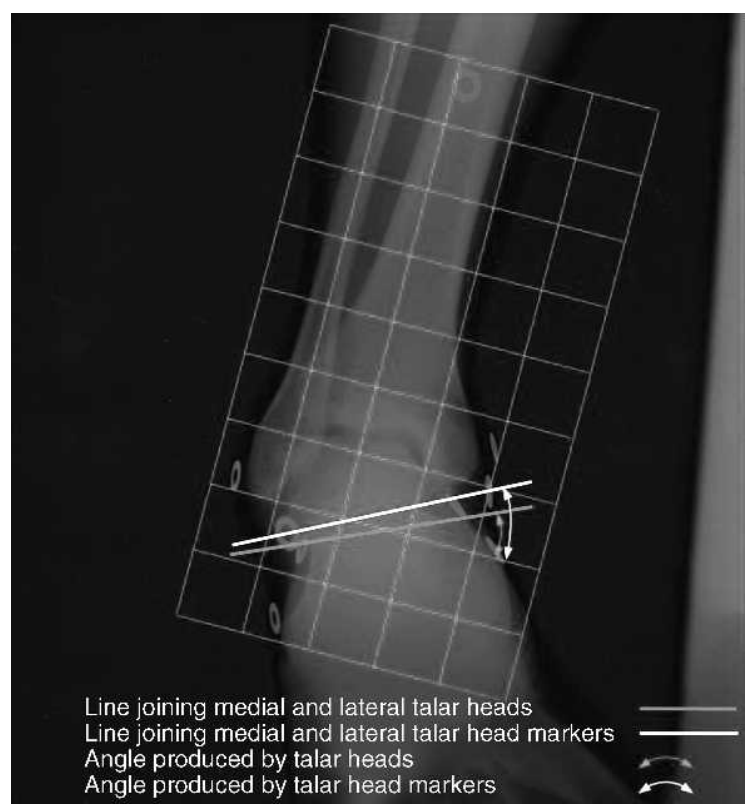

Figure 3. Example of the method of assessing the effect of marker displacement on angular measurements (frontal plane). set of planar definitions. On each radiograph, lines were then drawn between each pair of markers and each pair of bony landmarks. The angle of each of these lines relative to the grid lines was then measured (Fig. 3). In all cases, the angles were measured using a protractor and were rounded to the nearest whole degree. Consequently, the impact of the skin movement on the angular measurements could be quantified.

\section{Results}

\section{Radiograph Orientation}

The relationship between the CODA marker separations and those derived from the radiographs was found to be strong, suggesting a close match between the orientation in which the radiographs were taken and the measurement framework of the CODA (Table 1). In the case of the pronated and supinated radiographs, the transverse plane data showed the weakest relationship.

\section{Marker Displacement}

The relative displacement of the markers with respect to their bony landmarks varied from 0.61 to $22.18 \mathrm{~mm}$ (Table 2 and Fig. 4).

\section{Effect of Skin Movement on Angular Measurements}

The angular distortion caused by the movement of the skin markers relative to the bony landmarks they represent was found to be only a $1^{\circ}$ underestimation in the sagittal and transverse planes and a $5^{\circ}$ overestimation in the frontal plane (Table 3).

\section{Discussion}

This investigation was the second stage in the development of a robust, noninvasive, in vitro method of quantifying subtalar joint kinematics. The marker placement model designed for this purpose and outlined by Birch and Deschamps ${ }^{20}$ required markers to be placed on the tibia, fibula, talus, and calcaneus. Whereas the locations of the calcaneal markers meant that they were placed on skin tightly bound to the underlying bone, the positions of the talar and malleolar markers meant that they were attached to skin less well bound. This investigation, therefore, focused on the skin movement at these locations. 
Table 1. Correlation Coefficients for CODA Measurements versus Radiograph Measurements

\begin{tabular}{|c|c|c|c|c|c|c|}
\hline & \multicolumn{3}{|c|}{ Pronated } & \multicolumn{3}{|c|}{ Supinated } \\
\hline & $\begin{array}{c}\text { YZ/ } \\
\text { Sagittal Plane }\end{array}$ & $\begin{array}{c}\mathrm{XY} / \\
\text { Transverse Plane }\end{array}$ & $\begin{array}{c}\mathrm{XZ/} \\
\text { Frontal Plane }\end{array}$ & $\begin{array}{c}\text { YZ/ } \\
\text { Sagittal Plane }\end{array}$ & $\begin{array}{c}\mathrm{XY} / \\
\text { Transverse Plane }\end{array}$ & $\begin{array}{c}\text { XZ/ } \\
\text { Frontal Plane }\end{array}$ \\
\hline Correlation coefficient & 0.892 & 0.887 & 0.971 & 0.952 & 0.820 & 0.960 \\
\hline
\end{tabular}

Table 2. Marker Axial Displacement Measurements After Correction

\begin{tabular}{lrrrrrrr}
\hline & \multicolumn{3}{c}{ Pronated } & & \multicolumn{3}{c}{ Supinated } \\
\cline { 2 - 3 } \cline { 6 - 7 } Marker Separation & \multicolumn{1}{c}{$\mathrm{x}$} & $\mathrm{y}$ & $\mathrm{z}$ & & $\mathrm{x}$ & $\mathrm{y}$ \\
\hline Lateral malleolus - lateral malleolar marker & 5.12 & -8.03 & 3.44 & & 2.40 & -15.08 & 2.90 \\
Medial malleolus - medial malleolar marker & -3.54 & -6.26 & 0.61 & & -1.97 & -7.66 & 8.09 \\
Lateral talar head - lateral talar head marker & 10.57 & 8.70 & 13.54 & & 4.44 & -2.93 & 4.63 \\
Medial talar head - medial talar head marker & -5.45 & -6.32 & 6.65 & & -8.96 & 5.03 & 22.18 \\
\hline
\end{tabular}

Note: Data are given in millimeters.

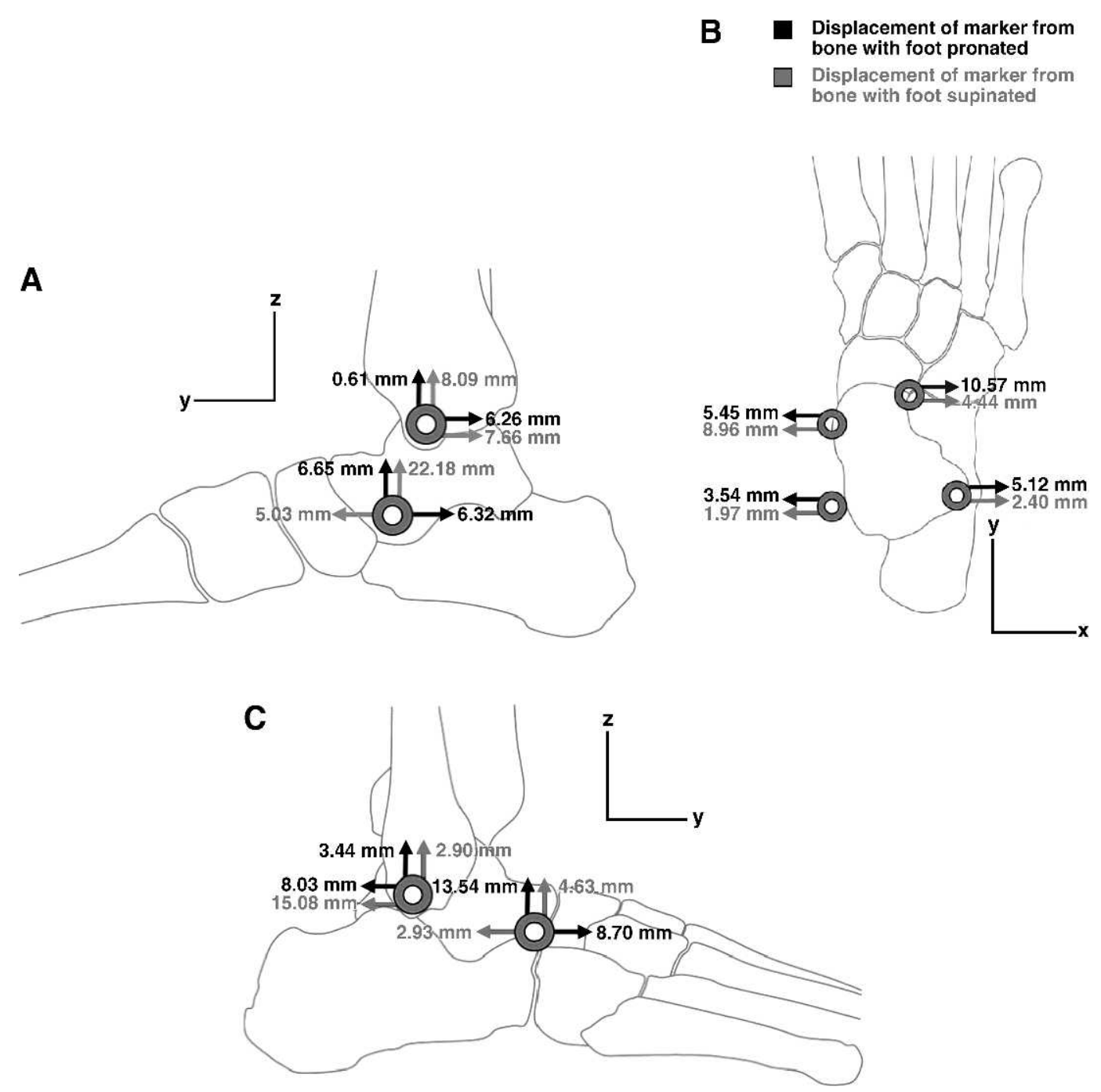

Figure 4. Movement of skin markers over bony landmarks in the $y$ and $z$ directions, medial $(A)$, in the $x$ direction, transverse $(B)$, and in the $y$ and $z$ directions, lateral $(C)$. 
Table 3. Frontal, Sagittal, and Transverse Plane Angular Movements Measured from the Radiographs

\begin{tabular}{|c|c|c|c|c|}
\hline & $\begin{array}{c}\text { Pronated } \\
\left({ }^{\circ}\right)\end{array}$ & $\begin{array}{l}\text { Supinated } \\
\left(^{\circ}\right)\end{array}$ & $\begin{array}{c}\text { Total Range } \\
\left({ }^{\circ}\right)\end{array}$ & Net Result \\
\hline \multicolumn{5}{|l|}{$\begin{array}{l}\text { Frontal plane angles measured from the transverse } \\
\text { plane to a line drawn between the: }\end{array}$} \\
\hline Lateral talar head and medial talar head & -4 & 20 & 24 & \multirow{2}{*}{$\begin{array}{l}5^{\circ} \text { overestimation of frontal plane } \\
\text { movement by the markers }\end{array}$} \\
\hline $\begin{array}{l}\text { Lateral talar head marker and medial talar head } \\
\text { marker }\end{array}$ & -2 & 27 & 29 & \\
\hline \multicolumn{5}{|l|}{$\begin{array}{l}\text { Sagittal plane angles measured from the transverse } \\
\text { plane to a line drawn between the: }\end{array}$} \\
\hline $\begin{array}{l}\text { Midpoint between lateral and medial talar heads } \\
\text { and midpoint between lateral and medial } \\
\text { malleoli }\end{array}$ & -26 & -50 & 24 & \multirow[t]{3}{*}{$\begin{array}{l}1^{\circ} \text { underestimation of frontal plane } \\
\text { movement by the markers }\end{array}$} \\
\hline $\begin{array}{l}\text { Midpoint between lateral and medial talar head } \\
\text { markers and midpoint between lateral and } \\
\text { medial malleolar markers }\end{array}$ & -8 & -31 & 23 & \\
\hline \multicolumn{4}{|l|}{$\begin{array}{l}\text { Transverse plane angles measured from the long } \\
\text { axis of the second metatarsal and a line drawn } \\
\text { between the: }\end{array}$} & \\
\hline Lateral talar head and medial talar head & 102 & 72 & 30 & \multirow{2}{*}{$\begin{array}{l}1^{\circ} \text { underestimation of frontal plane } \\
\text { movement by the markers }\end{array}$} \\
\hline $\begin{array}{l}\text { Lateral talar head marker and medial talar head } \\
\text { marker }\end{array}$ & 99 & 70 & 29 & \\
\hline
\end{tabular}

The skin movement occurring at the four anatomical locations was clearly not uniform. The movement of the malleolar markers appears to be less than that of the talar head markers. This is perhaps to be expected because the lateral and medial malleolar markers lie close to the bony landmarks, with only a thin layer of skin and subcutaneous soft tissue separating them. This can be seen from the $\mathrm{x}$-axis displacement, which showed there to be a maximum of only $5.12 \mathrm{~mm}$ between the lateral malleolus and the lateral malleolar marker. The same measurement for the lateral side of the head of the talus was $10.57 \mathrm{~mm}$. This suggests that the greater the amount of soft tissue separating a marker from its bony landmark, the greater the possible movement. However, the displacement that occurred was not uniform for all three planes. This variation was possibly the result of the nature of the underlying soft tissue. The large movement of the lateral talus marker anteriorly and the medial talus marker posteriorly and inferiorly could be the result of the effect of the tendons crossing the ankle and subtalar joint. As the foot was pulled into a pronated position, these tendons would have been placed under tension. As a result, they would have lifted from the underlying bone until stopped by the retinaculum and fascia. This would have lifted the markers away from the underlying bones.
The implications of this movement on the outcome of any investigation into the relative movements of the talus and calcaneus are more complex. Although the markers clearly move over the bones, the actual effect on any system's ability to measure the movement that occurs between the talus and the calcaneus depends on the effect of this movement on the intersegmental angle calculations. Although two markers may undergo substantial movement, the angle created by a line drawn between them may not change at all. Conversely, a relatively small movement of a marker away from its bony landmark could substantially affect the system's ability to assess the orientation of the underlying bones. The critical factor is the movement of the two markers relative to each other. To investigate this, the second set of measurements was taken from the radiographs. These showed the angular distortion caused by the movement of the skin markers relative to the bony landmarks they represent to be only a $1^{\circ}$ underestimation in the sagittal and transverse planes and a $5^{\circ}$ overestimation in the frontal plane. This variation in the degree to which the angular measurements were affected by the skin movement was perhaps to be expected in view of the findings from the first part of the study. However, there are a variety of factors that could complicate this finding. In this investigation, only the effects of pronation and supination were 
assessed. The effects of ankle joint plantarflexion and dorsiflexion could result in slightly different outcomes. Furthermore, the positioning of the foot was undertaken actively by the subject, producing muscle and tendon movements beneath the skin. Had the positioning of the foot been undertaken passively, again, slightly different outcomes may have been produced.

\section{Conclusions}

The movement of skin-mounted markers over the bony landmarks varies from location to location. This differential movement could be exacerbated by the nature and activity of the structures underlying the area of skin in question. Although the outcome of the measurement of the actual movement of the individual markers relative to the bony landmarks showed substantial movement, the effect that the movement would have on estimations of the angular relationship was substantially less than might have been expected.

The prediction of the effect of absolute movement on the estimation of angular movements of body segments defined by combinations of markers is difficult to predict on the basis of measurements of the absolute movement of individual markers. The results of this investigation suggest that for this subject, skin-mounted markers could be used to assess subtalar joint motion.

Acknowledgment: Simon Costain of the Gait and Posture Centre, London, England, for his help during the data collection phase of this work.

Financial Disclosure: None reported.

Conflict of Interest: None reported.

\section{References}

1. BAKer R, RobB J: Foot models for clinical gait analysis. Gait Posture 23: 399, 2006.

2. Rankine L, Long J, CANSECo K, ET AL: Multisegment foot modeling: a review. Crit Rev Biomed Eng 36: 127, 2008.

3. Davis RB, Ounpuu S, Tyburski D, Et AL: A gait analysis data collection and reduction technique. Hum Mov Sci 10: 575,1991 .

4. Kadaba MP, Ramakrishnan HK, Wootten ME: Measurement of lower extremity kinematics during level waking. J Orthop Res 8: 383, 1990.

5. Brown KM, Bursey DE, Arneson LJ, et al: Consideration of digitization precision when building local coordinate axes for a foot model. J Biomech 42: 1263, 2009.
6. Nester CJ, Lui AM, Howard D, ET AL: In vitro study of foot kinematics using a dynamic walking cadaver model. J Biomech 40: 1927, 2007.

7. Nester N, Jones RK, Lui A, ET AL: Foot kinematics during walking measured using bone and surface mounted markers. J Biomech 40: 3412, 2007.

8. Okita N, Meyers SA, Challis JH, et al: An objective evaluation of a segmented foot model. Gait Posture 30: 27, 2009.

9. AlexANder EJ, ANDRIACChi TP: Correcting for deformation in skin-based marker systems. J Biomech 34: 355, 2001.

10. Cappozoo A, Catini F, Leardini A, et al: Position and orientation in space of bones during movement: experimental artefacts. Clin Biomech 11: 90, 1996.

11. Leardini A, Chiari L, Della Croce U, et al: Human movement analysis using stereophotogrammetry: part 3. Soft tissue artifact assessment and compensation. Gait Posture 21: 212, 2005.

12. Lu TW, O'ConNer JJ: Bone position estimation from skin marker co-ordinates using global optimization with joint constraints. J Biomech 32: 129, 1999.

13. Manal K, McClay I, Richards J, et al: Knee moment profiles during walking: errors due to soft tissue movement of the shank and the influence of the reference coordinate system. Gait Posture 15: 10, 2002.

14. Reinschmidt C, Van der bogert AJ, Lundberg A, et AL: Tibiofemoral and tibiocalcaneal motion during walking: external vs skeletal markers. Gait Posture 6: 98, 1997.

15. Karlsson D, Tranberg R: On skin movement artifactresonation frequencies of skin markers attached to the leg. Hum Mov Sci 18: 627, 1999.

16. Lucchetti L, Cappozzo A, Cappello A, et AL: Skin movement artefact assessment and compensation in the estimation of knee-joint kinematics. J Biomech 31: 977, 1998.

17. Holden JP, Orsini S, Lohmann Siegel K, et al: Surface movement errors in shank kinematics and knee kinematics during gait. Gait Posture 5: 217, 1997.

18. Karlsson D, LundBerg A: "Accuracy estimation of kinematic data derived from bone anchored external markers," in Proceeding of the Third International Symposium on 3-D Analysis of Human Motion, p 27, Hasselbacken Conference Center, Stockholm, Sweden, 1994.

19. Westblad P, Hashimoto T, Winson I, et al: Differences in ankle-joint complex motion during the stance phase of walking as measured by superficial and bone anchored markers. Foot Ankle Int 23: 856, 2002.

20. BiRch I, Deschamps K: The in vitro reliability of the CODA MPX30 as the basis for a method of assessing the in vivo motion of the subtalar joint. JAPMA 101: 400, 2011.

21. Wu G, Siegler S, Allard P, ET AL: ISB recommendation on definitions of joint coordinate system of various joints for the reporting of human joint motion: part I. Ankle, hip, and spine. J Biomech 5: 543, 2002. 